\title{
Trends in the use of antibiotics among Korean children
}

\author{
Young June Choe, MD, PhD', Ju-Young Shin, PhD² \\ ${ }^{1}$ Division of Pediatric Infectious Diseases, The Warren Alpert Medical School of Brown University, Providence, RI, USA, ${ }^{2}$ School of Pharmacy, Sungkyunkwan \\ University, Suwon, Korea
}

Inappropriate antibiotic use is the most important factor causing increased bacterial resistance to antibiotics, thus affecting patient outcomes. Multidrug-resistant bacteria have become a serious public health threat, causing significant morbidity and mortality worldwide. In Korea, the burden of antibioticresistant bacteria has become an important public health issue. There is increasing evidence of overuse and misuse of antibiotics in Korea, as observed in cohorts with large sample sizes. Antibiotic use among children should receive particular attention because of the frequency of community-associated infections among this population and the elevated risk of transmission. Recent studies from Korea have demonstrated that the use of broad-spectrum antibiotics, either for inpatient or outpatient treatment, has increased among many age groups, especially children. In this review, we aim to describe the patterns of antibiotic prescription and evaluate recent trends in antibiotic use among children. Coordinated efforts toward communication and education in order to address misunderstandings regarding antibiotic use, involving interprofessional antimicrobial stewardship programs, are required in the near future.

Key words: Antibiotic, Antimicrobial, Consumption, Use, Resistance

\section{Introduction}

Infections caused by antibiotic-resistant pathogens cause substantial morbidity and mortality globally. ${ }^{1)}$ Inappropriate use and overuse of antibiotics are the most important factors causing increased bacterial resistance, thus affecting patient outcomes and the spread of infections caused by multidrug-resistant organisms. ${ }^{2)}$ Antibiotics can be overprescribed in everyday clinical practice; for instance, antibiotics may be prescribed for conditions in which antibiotics are not indicated, such as respiratory viral infections. ${ }^{3)}$ In addition to the use of unnecessary antibiotics, the inappropriate use of broad-spectrum agents when narrowerspectrum agents are indicated is common. ${ }^{4}$

In Korea, antibiotic overuse and the burden of antibiotic resistance have become important public health issues in recent years. Antibiotic use among children should receive particular attention because of the frequency of community-associated infections among this age group, their longer life expectancies, and the elevated risk of exposure to antibiotic-resistant pathogens in cases of drug control. ${ }^{5)}$ Professional societies have encouraged judicious antibiotic use, focusing on decreasing the inappropriate use of broad-spectrum agents in both inpatient and outpatient settings. ${ }^{6}$ However, descriptive data to estimate antibiotic use is important for evaluating the changes driven by policies. In this review, we aim to describe the patterns of antibiotic prescription and to evaluate recent trends in antibiotic use among children in Korea.
Corresponding author: Ju-Young Shin, PhD School of Pharmacy, Sungkyunkwan University, 2066 Seobu-ro, Jangan-gu, Suwon 16419, Korea Tel: +82-31-290-7702

Fax: +82-31-292-8800

E-mail: shin.jy@skku.edu https://orcid.org/0000-0003-1010-7525

Received: 19 November, 2018

Revised: 21 February, 2019

Accepted: 4 March, 2019
Copyright ( 2019 by The Korean Pediatric Society

This is an open-access article distributed under the terms of the Creative Commons Attribution NonCommercial License (http://creativecommons.org/ licenses/by-nc/4.0/) which permits unrestricted noncommercial use, distribution, and reproduction in any medium, provided the original work is properly cited. 


\section{Concerns of antibiotic resistance}

According to World Health Organization (WHO) data from 2014, the prevalence of resistant organisms is considerably high in Korea; the resistance rate of Staphylococcus aureus to methicillin ranges from 57.7\% to 73.0\%, while that of Escherichia coli to third-generation cephalosporin ranges from $23.9 \%$ to $28.0 \%{ }^{7)}$ The prevalence of antibiotic resistance in Korea varies across pathogens and collection periods, but is comparable to that in neighboring Asian countries. Fig. 1 depicts a comparison of the general prevalence of antibiotic resistance in Korea and other Asian countries. Among clinical isolates of Streptococcus pneumoniae from 11 Asian countries, between 2000 and 2001, isolates from Korea showed the second highest prevalence of penicillin resistance (54.8\%), after those from Vietnam (71.4\%), followed by isolates from Hong Kong (43.2\%) and Taiwan $(38.6 \%){ }^{8)}$ Among E. coli isolates from urinary tract infections, 20\% of the isolates from Korea were extended-spectrum beta lactamaseproducers, with the country ranking seventh in this regard among Asian countries. ${ }^{9)}$ The prevalence of methicillin resistance in hospitalacquired $S$. aureus isolates was also high in Korea, at 77.6\% after only Sri Lanka (86.5\%), and followed by that in Vietnam (74.1\%),
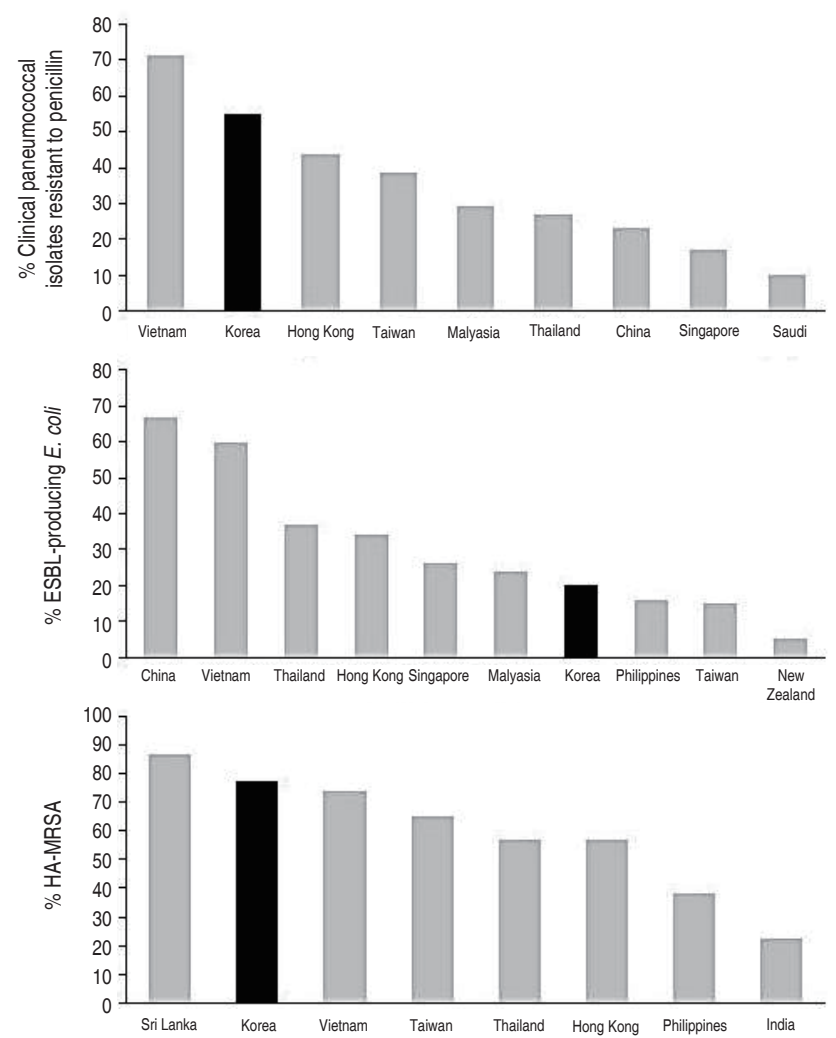

Fig. 1. Antibiotic resistance among Streptococcus pneumoniae, Escherichia coli, and Staphylococcus aureus by country; World Health Organization, 2014. ESBL, extended-spectrum beta-lactamase; HAMRSA, healthcare-associated methicillin resistant Staphylococcus aureus. Adapted from World Health Organization (WHO). ${ }^{11}$
Taiwan (65.0\%), and Thailand (57.0\%). ${ }^{10)}$

\section{Global antibiotic consumption}

Globally, antibiotic consumption has been low in high-income countries, whereas it has been disproportionally high in lowand middle-income countries. One study that reviewed trends in antibiotic consumption in 71 countries between 2000 and 2010 showed that antibiotic consumption was stable or had moderately decreased in high-income countries. ${ }^{11)}$ According to a more recent study that extended the analysis to 2015, the consumption rates for cephalosporins, quinolones, and macrolides decreased in highincome countries and increased in low- and middle-income countries. ${ }^{12)}$ In both studies, Korea showed increasing trends of antibiotic consumption and had the highest consumption rate among highincome countries. Cross-sectional data presented in 2014 by the Organisation for Economic Co-operation and Development (OECD) suggested that the rate of antibiotic consumption in Korea is 31.7 defined daily doses per 1,000 inhabitants/day (DID), while the rate of average use in OECD countries is 20.5 DID (Fig. 2). ${ }^{13)}$ Countries with comparable population sizes and economies, such as Spain and Italy, had lower DID than did Korea, at 21.6 and 27.8 DID, respectively.

A brief summary combining OECD data on antibiotic consumption with WHO data on antibiotic resistance rates is provided in Fig. 3. The prevalence of resistant organisms by proportion is generally correlated with the magnitude of antibiotic consumption in different OECD countries. Small or moderate correlation was noted for $E$. coli resistance to fluoroquinolones $\left(R^{2}=0.289\right)$, Klebsiella pneumoniae resistance to third-generation cephalosporins $\left(R^{2}=0.220\right)$, and methicillin-resistant Staphylococcus aureus (MRSA) $\left(R^{2}=0.316\right)$. Korea was largely at the higher end for both antibiotic consumption and prevalence of resistant organisms.

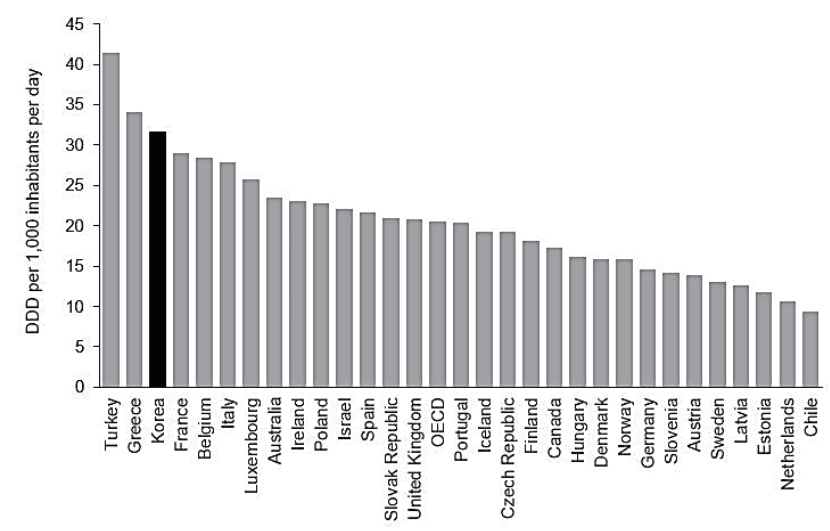

Fig. 2. Average antibiotic consumption in Organisation for Economic Cooperation and Development (OECD) member countries, 2005-2016. DDD, defined daily dose. Adapted from Organisation for Economic Cooperation and Development. ${ }^{13)}$ 


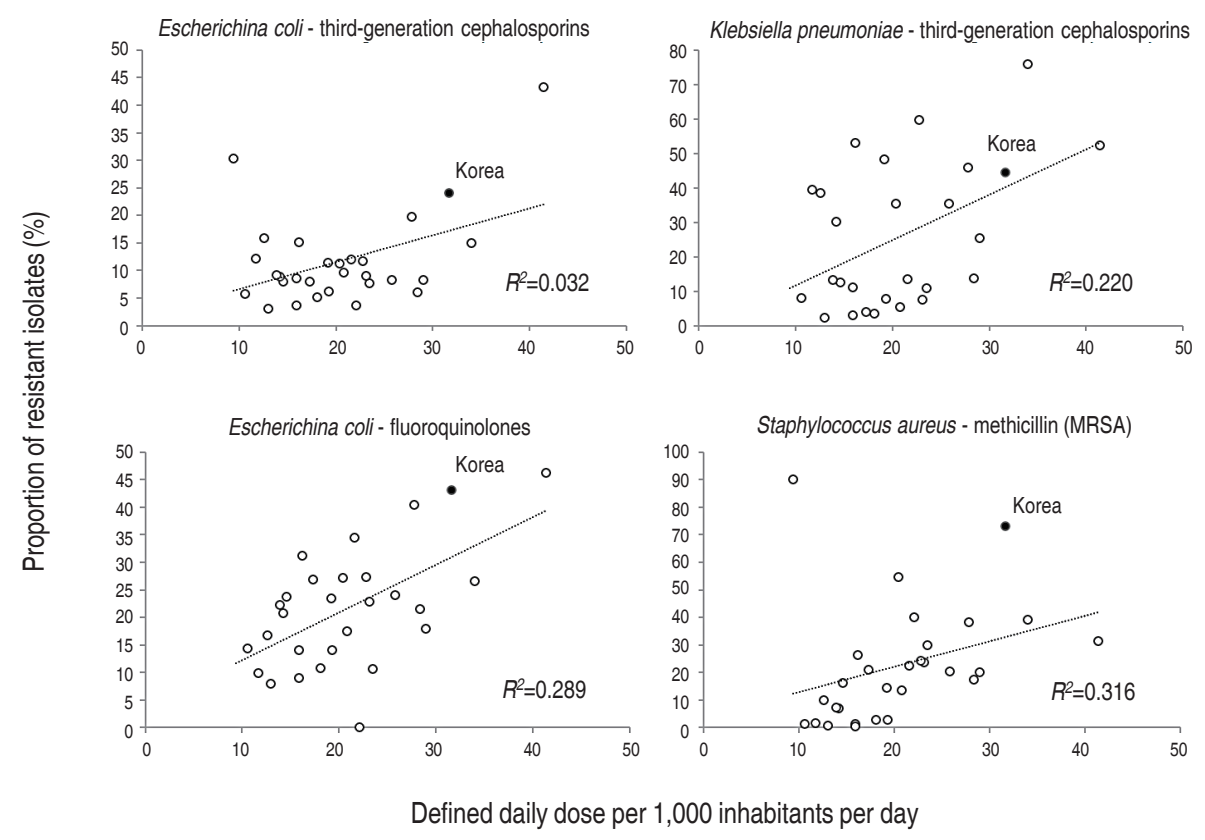

Fig. 3. Correlation between antibiotic consumption (2005-2016) and antibiotic resistance (2014). Adapted from references World Health Organization $(\mathrm{WHO})^{1)}$ and Organisation for Economic Co-operation and Development. $^{13)}$

Globally, antibiotics are the most commonly prescribed class of medication among children. A study from the United States (US) that used an outpatient prescription drug utilization program showed that amoxicillin was the most frequently dispensed prescription for infants and children in 2010 ( $n=18,292,768)$, followed by azithromycin $(n=10.171,046)$, albuterol $(n=7,343,063)$, and amoxicillinclavulanate $(n=4,454,926) .{ }^{14)}$ Systemic antibiotics were the most frequently dispensed medications for children, accounting for 24\% to 27\% of all prescriptions between 2002 and 2010; however, the number of prescriptions decreased by 14\% during this period. Cefdinir-a second-generation cephalosporin-and cephalexin-a first-generation cephalosporin-were the only prescribed cephalosporins studied in this investigation.

Over the last two decades, professional societies such as the American Academy of Pediatrics have addressed the issue of antibiotic overuse and the development of drug-resistant organisms by educating parents and promoting evidence-based guidelines among practitioners. ${ }^{15)}$ Their efforts have been supported by the government: former US president Barack Obama released an executive order on combating antibiotic-resistant bacteria. ${ }^{16)}$ A variety of initiatives from the medical community also promote the appropriate use of antimicrobial drugs and the prevention of antibiotic-resistant infections.

\section{The Korean context}

The recent trends in Korean antibiotic consumption at the national level have been evaluated in a few studies. The first study used National Health Insurance claims data collected through the Health Insurance Review and Assessment (HIRA) service to quantify and analyze patterns of total systemic antibiotic prescriptions filed in Korea from 2008 to 2012. ${ }^{17)}$ The results showed that the total consumption of antibiotics increased from 21.68 DID in 2008 to 23.12 DID in 2012. Penicillins were the most commonly used antibiotics (4.52 DID), followed by second-generation cephalosporins (4.47 DID) and macrolides (3.32 DID). There was a significant increase in the consumption of third-generation cephalosporins, carbapenems, and glycopeptides $(P<0.001)$. The study also investigated the differences in these trends among age groups. Compared to adolescents and adults, children had higher rates of penicillin, macrolide, and third-generation cephalosporin consumption (2.2-, 2.0-, and 2.6 fold greater, respectively). The second study also used HIRA claims data for a similar study period (2007-2014), but included additional information on trend changes for antibiotic classes in different age groups. ${ }^{18)}$ Children aged younger than 6 years had the highest level of use (59.21 DID) in 2014, and showed an increasing trend each year, consistent with previous findings. The prescription rate of extended-spectrum penicillins and third- and fourth-generation cephalosporins showed an increasing trend, while the prescription rate of first- and second-generation cephalosporins seemed to have declined (Fig. 4). 
A cross-national analysis was conducted from cohorts from 6 countries, including Germany, Italy, Korea, Norway, Spain, and the US, between 2008 and 2012. ${ }^{19)}$ This study showed that Korea had the highest rate of antimicrobial consumption among children, with 3.41 prescribed courses per child per year during the first 2 years of life, as compared to 1.4-1.6 in Italy, 1.5 in Spain, 1.1 in the US, 1.0 in

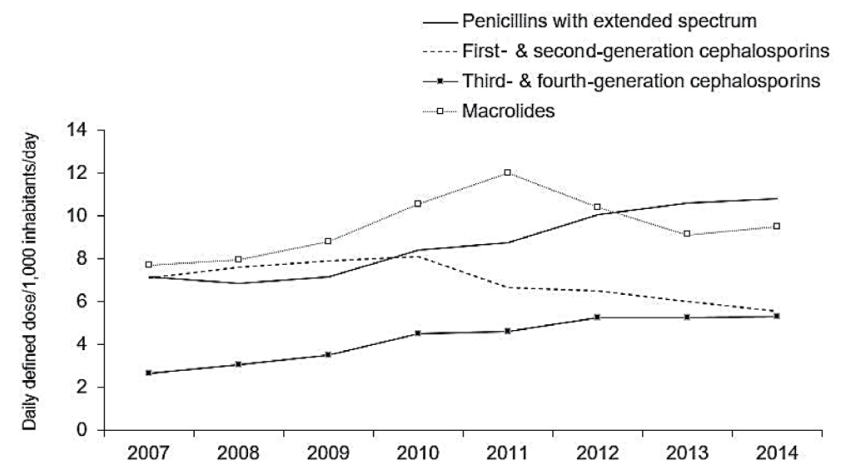

Fig. 4. Trend of antibiotic consumption in children aged $\leq 6$ years in Korea, 2007-2014. Adapted from Park et al., PLoS One 2017;12: e0177435. ${ }^{18)}$

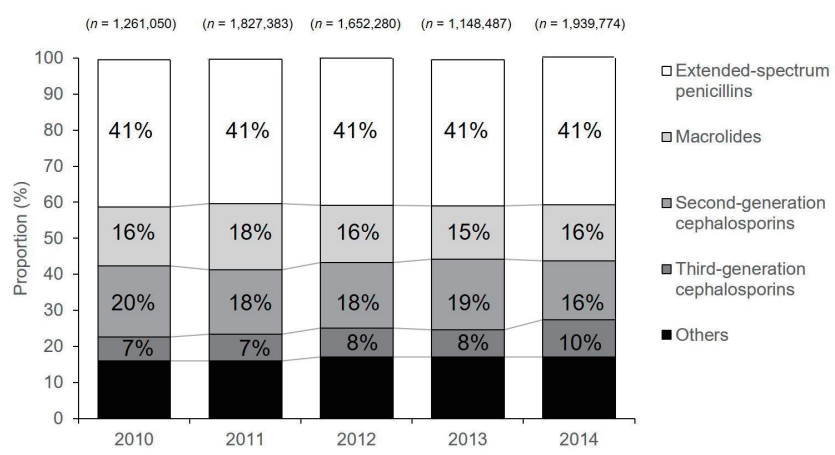

Fig. 5. Proportion of antibiotic prescriptions in Korean children in 20102014. Adapted from Song et al. Pediatr Int 2018;60:803-10. ${ }^{20)}$
Germany, and 0.5 in Norway. The finding suggests that an average toddler in Korea is prescribed antibiotics at a prescription rate 7.5 fold higher than that in Norway. Moreover, higher proportions of broad-spectrum agents are used in Korea. The study found that among all pediatric antimicrobial prescriptions in Korea, 9.8\% were first-line penicillins (likely amoxicillin), whereas in Norway and Germany, 64.8\% and 39.6\% were first-line penicillins, respectively. Multiple factors may affect the choice of antibiotics in the treatment of children with possible bacterial infection. In Korea, which has higher resistance rates, healthcare providers are more inclined to prescribe antibiotics with broader coverage. However, this practice exerts constant selective pressure due to broad-spectrum antibiotics, which may cause a further increase in the resistance rate.

One recent study that used National Patient Sample data provided by the HIRA mainly focused on the pediatric population. ${ }^{20)}$ The data was extracted from claims for 1.4 million patients per year (3\%), using gender- and age-stratified random sampling, and included the generic name, prescription date, dosage, duration, and route of administration of the drug. Diagnoses were recorded according to the International Classification of Diseases, Tenth Revision. The study population consisted of patients aged 2-17 years, for whom antibiotics were prescribed in the outpatient setting. In total, 129 systemic antibiotics that were coded as J01 (according to the WHO Anatomical Therapeutic Chemical classification system) were included. Antibiotics were grouped into 25 different classes according to their mode of action. The antibiotic rate was calculated by dividing the number of antibiotic prescriptions by the number of all outpatient prescriptions per year, categorized by age group. The frequency and percentage of antibiotic prescriptions were calculated for each antibiotic class. The defined daily dose was calculated for individual antibiotics. Between 2010 and 2014, in total, 7,261,176 antibiotic prescriptions were provided to 1,039,756 patients. Most of these prescriptions (91.2\%) were for the treatment of respiratory

Table 1. Annual antibiotic prescription patterns by antibiotic class represented using the DDD per 1,000 inhabitants per day among pediatric outpatients in Korea in 2010-2014

\begin{tabular}{|c|c|c|c|c|c|c|c|}
\hline \multirow{2}{*}{ Individual antibiotics } & \multirow{2}{*}{ DDD/1,000 patients/day } & \multirow{2}{*}{$\%$ DDD } & \multicolumn{5}{|c|}{ Annual DDD/1,000 patients/day } \\
\hline & & & 2010 & 2011 & 2012 & 2013 & 2014 \\
\hline \multicolumn{8}{|l|}{ Penicillins } \\
\hline Amoxicillin & 3.51 & 2.4 & 3.51 & 4.59 & 2.68 & 2.44 & 4.15 \\
\hline Amoxicillin/clavulanate & 67.40 & 45.1 & 68.96 & 94.62 & 49.01 & 44.03 & 75.26 \\
\hline \multicolumn{8}{|l|}{ Macrolides } \\
\hline Clarithromycin & 26.14 & 17.50 & 25.03 & 42.37 & 17.97 & 14.85 & 27.28 \\
\hline Roxithromycin & 5.46 & 5.46 & 6.58 & 7.91 & 3.81 & 3.44 & 4.77 \\
\hline \multicolumn{8}{|l|}{ Cephalosporines } \\
\hline Cefaclor & 13.77 & 9.2 & 16.03 & 19.26 & 9.82 & 8.89 & 13.25 \\
\hline Cefuroxime & 3.73 & 2.5 & 5.26 & 5.38 & 2.31 & 1.98 & 3.01 \\
\hline Cefpodoxime & 16.78 & 11.2 & 13.14 & 22.23 & 12.57 & 10.72 & 25.82 \\
\hline
\end{tabular}

$\mathrm{DDD}$, defined daily dose.

Adapted from Song et al. Pediatr Int 2018;60:803-10. ${ }^{20)}$ 
illnesses. The most frequent primary diagnosis was acute upper respiratory infection (24.4\%), followed by acute bronchitis (22.2\%). The antibiotic prescription rate was the highest for patients aged 2-6 years, comprising $84.0 \%$ of all outpatient prescriptions for that age group in 2014. The antibiotic prescription rate for all pediatric patients increased from 34.8\% in 2010 to 70.4\% in 2014. Among drug classes, extended-spectrum penicillins were the most commonly prescribed; however, the proportion of prescriptions for the class remained constant at 40.0\%-41.0\% between 2010 and 2014 (Fig. 5). Interestingly, the proportion of prescriptions for thirdgeneration cephalosporins increased from 6.6\% in 2010 to 10.3\% in 2014, resulting in a relative difference of 55.7\% (95\% confidence interval, 55.2-56.2), while the percentages of prescriptions for firstand second-generation cephalosporins decreased over the same period. Amoxicillin-clavulanate was the most commonly used drug, and its use increased by 9.0\% between 2010 and 2014 (Table 1). Unlike a previous study, this study showed that amoxicillinclavulanate was more commonly prescribed than amoxicillin, which is the first-line drug of choice for many community-acquired respiratory tract infections. The reason for the increased use of third-generation cephalosporins is unclear. A concurrent decline in second-generation cephalosporins suggests that this class of drugs has been replaced by third-generation cephalosporins, without any clear explanation. However, this change in the pattern of drug prescriptions for children may have led to changes in overall prescription patterns among all age groups in Korea during the previous decade.

\section{Conclusions}

Clearly, antibiotic consumption among Korean children is considerably high. In addition to antibiotic-related issues, high rates of polypharmacy have been frequently observed in children. One study demonstrated a higher rate of polypharmacy in a pediatric population aged 1-9 years than in an older population (19.4\% vs. $4.5 \%$ in $2002-2003,12.3 \%$ vs. $3.3 \%$ in $2012-2013) .{ }^{211}$ However, there was a decreasing trend in the prevalence of polypharmacy, from $65.3 \%$ in 2002 to $46.6 \%$ in 2013, which likely reflects the policy-driven change since the early 2000s that stemmed from quality assessment and a drug utilization review by the HIRA service. ${ }^{7)}$ These government-driven policies have played a pivotal role in controlling antibiotic use in Korea in recent decades. However, coordinated efforts toward communication with and education of parents in order to address misunderstandings regarding antibiotic use, involving interprofessional antimicrobial stewardship programs, are required in the near future.

\section{Conflicts of interest}

No potential conflict of interest relevant to this article was reported.

\section{References}

1. World Health Organization. Antimicrobial resistance: global report on surveillance 2014 [Internet]. Geneva (Switzerland): World Health Organization; 2014 [cited 2018 0ct 30]. Available from: http://www. who.int/drugresistance/documents/surveillancereport/en/.

2. Fleming-Dutra KE, Hersh AL, Shapiro DJ, Bartoces M, Enns EA, File $\mathrm{TM} \mathrm{Jr}$, et al. Prevalence of inappropriate antibiotic prescriptions among us ambulatory care visits, 2010-2011. JAMA 2016;315:186473.

3. Arroll B, Kenealy T. Antibiotics for the common cold. Cochrane Database Syst Rev. 2002;(3):CD000247.

4. Lindbaek M. Broad-spectrum antibiotics gave no clinical benefit and more adverse effects than narrow-spectrum antibiotics in treating acute respiratory tract infections in US children. BMJ Evid Based Med 2018 Oct 25 [Epub]. pii: bmjebm-2018-110938. https://doi.org/ 10.1136/bmjebm-2018-110938.

5. Patel SV, Vergnano S. The impact of paediatric antimicrobial stewardship programmes on patient outcomes. Curr Opin Infect Dis 2018; 31:216-23.

6. Society for Healthcare Epidemiology of America; Infectious Diseases Society of America; Pediatric Infectious Diseases Society. Policy statement on antimicrobial stewardship by the Society for Healthcare Epidemiology of America (SHEA), the Infectious Diseases Society of America (IDSA), and the Pediatric Infectious Diseases Society (PIDS). Infect Control Hosp Epidemiol 2012;33:322-7.

7. Kim BN, Kim HB, Oh MD. Antibiotic control policies in South Korea, 2000-2013. Infect Chemother 2016;48:151-9.

8. Song JH, Jung SI, Ko KS, Kim NY, Son JS, Chang HH, et al. High prevalence of antimicrobial resistance among clinical Streptococcus pneumoniae isolates in Asia (an ANSORP study). Antimicrob Agents Chemother 2004;48:2101-7.

9. Lu PL, Liu YC, Toh HS, Lee YL, Liu YM, Ho CM, et al. Epidemiology and antimicrobial susceptibility profiles of Gram-negative bacteria causing urinary tract infections in the Asia-Pacific region: 2009-2010 results from the Study for Monitoring Antimicrobial Resistance Trends (SMART). Int J Antimicrob Agents 2012;40 Suppl:S37-43.

10. Song JH, Hsueh PR, Chung DR, Ko KS, Kang CI, Peck KR, et al. Spread of methicillin-resistant Staphylococcus aureus between the community and the hospitals in Asian countries: an ANSORP study. J Antimicrob Chemother 2011;66:1061-9.

11. Van Boeckel TP, Gandra S, Ashok A, Caudron Q, Grenfell BT, Levin SA, et al. Global antibiotic consumption 2000 to 2010: an analysis of national pharmaceutical sales data. Lancet Infect Dis 2014;14:742-50.

12. Klein EY, Van Boeckel TP, Martinez EM, Pant S, Gandra S, Levin SA, et al. Global increase and geographic convergence in antibiotic consumption between 2000 and 2015. Proc Natl Acad Sci U S A 2018;115:E3463-70.

13. Organisation for Economic Co-operation and Development. Antibiotic consumption levels, 2005-2016 [Internet]. Paris: Organisation for Economic Co-operation and Development; [2018 Oct 30]. Available from: http://www.oecd.org/els/health-systems/antimicrobialresistance.htm.

14. Chai G, Governale L, McMahon AW, Trinidad JP, Staffa J, Murphy D. 
Trends of outpatient prescription drug utilization in US children, 2002-2010. Pediatrics 2012;130:23-31.

15. Hersh AL, Jackson MA, Hicks LA; American Academy of Pediatrics Committee on Infectious Diseases. Principles of judicious antibiotic prescribing for upper respiratory tract infections in pediatrics. Pediatrics 2013;132:1146-54.

16. White House. Executive order combating antibioitc resistant bacteria [Internet]. Washington, DC: White House; 2014 Sep [2018 0ct 30]. Available from: http://www.whitehouse.gov/the-press-office/2014/ 09/18/executive-order-combating-antibiotic-resistant-bacteria.

17. Yoon YK, Park GC, An H, Chun BC, Sohn JW, Kim MJ. Trends of antibiotic consumption in Korea according to national reimbursement data (2008-2012): a population-based epidemiologic study. Medicine
(Baltimore) 2015;94:e2100.

18. Park J, Han E, Lee SO, Kim DS. Antibiotic use in South Korea from 2007 to 2014: A health insurance database-generated time series analysis. PLoS One 2017;12:e0177435.

19. Youngster I, Avorn J, Belleudi V, Cantarutti A, Díez-Domingo J, Kirchmayer U, et al. Antibiotic use in children - a cross-national analysis of 6 countries. J Pediatr 2017;182:239-44.e1.

20. Song I, Park SJ, Byun SJ, Choe YJ, Shin JY. Increased use of thirdgeneration cephalosporin antibiotics in the outpatient setting in Korean children and adolescents. Pediatr Int 2018;60:803-10.

21. Baek YH, Shin JY. Trends in polypharmacy over 12 years and changes in its social gradients in South Korea. PLoS One 2018;13: e0204018. 